\title{
Intersection Properties of Balls in Banach Spaces
}

\author{
C. R. Jayanarayanan \\ Stat-Math Unit, Indian Statistical Institute, R. V. College Post, Bangalore 560059, India \\ Correspondence should be addressed to C. R. Jayanarayanan; crjayan@gmail.com
}

Received 29 May 2013; Revised 25 July 2013; Accepted 20 August 2013

Academic Editor: Miguel Martin

Copyright (c) 2013 C. R. Jayanarayanan. This is an open access article distributed under the Creative Commons Attribution License, which permits unrestricted use, distribution, and reproduction in any medium, provided the original work is properly cited.

\begin{abstract}
We introduce a weaker notion of central subspace called almost central subspace, and we study Banach spaces that belong to the class (GC), introduced by Veselý (1997). In particular, we prove that if $Y$ is an almost central subspace of a Banach space $X$ such that $Y$ is in the class (GC), then $Y$ is a central subspace of $X^{* *}$. We also prove that if $Y$ is a semi $M$-ideal in a Banach space $X$ such that $Y^{\perp \perp}$ is an almost central subspace of $X^{* *}$, then $Y$ is an $M$-ideal in $X$. Certain stability results for quotient spaces, injective tensor product spaces, and polyhedral direct sums of Banach spaces are also derived.
\end{abstract}

\section{Introduction}

In [1], Veselý studied a new class of Banach spaces, namely, the class (GC), which were defined in terms of the existence of weighted Chebyshev centers (see below for definition and details). In the same paper, he characterized such spaces using intersection properties of balls. In [2], Bandyopadhyay and Rao considered some general results about the class (GC) by introducing a new class of subspaces called "central subspaces" of Banach spaces. Using this concept, they characterized the class (GC) and produced several examples of Banach spaces which belong to the class (GC) (see [2] for details). In this paper, we introduce and study a weaker notion of central subspace called almost central subspace (see Section 2 for definition and details). Using this concept, we obtain some new results about the class (GC) and also about some of the other types of intersection properties of balls studied in the literature.

For a Banach space $X$, we denote by $B_{X}(x, r)$ the closed ball in $X$ of radius $r>0$ around $x \in X$ and by $B_{X}$ the closed unit ball of $X$. In this paper, we restrict ourselves to real scalars and all subspaces we consider are assumed to be closed. Under the canonical embedding, we will consider $X$ as a subspace of $X^{* *}$. Also, if a Banach space $Y$ is isometric to a subspace of the Banach space $X$, then, without loss of generality, we will consider $Y$ as a subspace of $X$. Our notations are otherwise standard. Any unexplained terminology can be found in [3].
We now recall the definition of the class (GC) from [1].

Definition 1. Let $X$ be a Banach space. Let $a_{1}, \ldots, a_{n} \in X$ and $\eta_{1}, \ldots, \eta_{n}>0$. Minimizers of the function $\phi: X \rightarrow \mathbb{R}$ defined by $\phi(x)=\max _{1 \leq i \leq n} \eta_{i}\left\|x-a_{i}\right\|$ are called weighted Chebyshev centers with the weight $\eta=\left(\eta_{1}, \ldots, \eta_{n}\right)$. Classical Chebyshev centers are the weighted Chebyshev centers with the weight $\eta=(1, \ldots, 1)$.

Theorem 2 (see [1, Theorem 2.7]). For a Banach space $X$ and $a_{1}, \ldots, a_{n} \in X$, the following assertions are equivalent.

(i) If $r_{1}, \ldots, r_{n}>0$ and $\bigcap_{i=1}^{n} B_{X^{* *}}\left(a_{i}, r_{i}\right) \neq \emptyset$, then $\bigcap_{i=1}^{n} B_{X}\left(a_{i}, r_{i}\right) \neq \emptyset$.

(ii) $a_{1}, \ldots, a_{n}$ admits weighted Chebyshev centers for all weights $\eta=\left(\eta_{1}, \ldots, \eta_{n}\right)$, where $\eta_{i}>0$ for all $i$.

Definition 3 (see [1, Definition 2.8]). One shall denote by (GC) the class of all Banach spaces $X$ such that for every positive integer $n$ and every $a_{1}, \ldots, a_{n} \in X$, one of the equivalent conditions (i) or (ii) of Theorem 2 is satisfied.

Next we recall the definition of a central subspace which generalizes the notion of (GC).

Definition 4 (see [2, Definition 2.1]). Let $X$ be a Banach space. One says that a subspace $Y \subseteq X$ is a central subspace of $X$ 
if every finite family of closed balls with centers in $Y$ that intersects in $X$ also intersects in $Y$.

Clearly $X \in(\mathrm{GC})$ if and only if $X$ is a central subspace of $X^{* *}$. It follows from [2, Proposition 2.2(a)] that $Y$ is a central subspace of a Banach space $X$ if and only if, for any finite set $\left\{y_{i}\right\}_{i=1}^{n} \subset Y$ and $x \in X$, there exists a $y \in Y$ such that $\left\|y-y_{i}\right\| \leq$ $\left\|x-y_{i}\right\|$ for $1 \leq i \leq n$.

An infinite version of central subspace called almost constrained subspace was investigated in $[4,5]$.

Definition 5. A subspace $Y$ of a Banach space $X$ is said to be an almost constrained (AC) subspace of $X$ if any family of closed balls centered at points of $Y$ that intersects in $X$ also intersects in $Y$.

We recall that a subspace $Y$ of a Banach space $X$ is called 1-complemented in $X$ if there exists a projection of norm one on $X$ with range $Y$. One can easily observe that 1-complemented subspaces are AC-subspaces, and hence they are also central subspaces. The notion of an "ideal," which is weaker than being a 1-complemented subspace, was introduced by Godefroy et al. in [6].

Definition 6. A subspace $Y$ of a Banach space $X$ is said to be an ideal in $X$ if $Y^{\perp}$ is the kernel of a norm one projection on $X^{*}$.

Clearly 1-complemented subspaces are ideals. Also, every Banach space is an ideal in its bidual. For, if $X$ is a Banach space, then the projection $P: X^{* * *} \rightarrow X^{* * *}$ defined by $P(\Lambda)=\left.\Lambda\right|_{X}$ is a projection of norm one with kernel $X^{\perp}$. It is well known that $c_{0}$ is an ideal in $\ell_{\infty}$ but it is not the range of a projection of norm one in $\ell_{\infty}$.

An important concept in the $M$-structure theory which is closely related to ball intersection properties is the well known concept called $M$-ideal (see [7] for details).

Definition 7. A projection $P$ on a Banach space $X$ is called an $M$-projection (L-projection) if $\|x\|=\max \{\|P x\|,\|x-P x\|\}$ $(\|x\|=\|P x\|+\|x-P x\|)$ for all $x \in X$. A subspace $Y$ of $X$ is called an $M$-summand ( $L$-summand) if it is the range of an $M$-projection ( $L$-projection). A subspace $Y$ of $X$ is called an $M$-ideal if $Y^{\perp}$ is an $L$-summand in $X^{*}$. For two Banach spaces $X$ and $Z$, one denotes by $X \bigoplus_{1} Z$ and $X \bigoplus_{\infty} Z$ the direct sum of $X$ and $Z$, equipped with the $\ell_{1}$-norm and supremum norm, respectively.

For $n \in \mathbb{N}$, we recall that a subspace $Y$ of a Banach space $X$ is said to have the (strong) $n$-ball property if, given $n$ closed balls $\left\{B_{X}\left(a_{i}, r_{i}\right)\right\}_{i=1}^{n}$ in $X$ such that $\bigcap_{i=1}^{n} B_{X}\left(a_{i}, r_{i}\right) \neq \emptyset$ and $Y \cap B_{X}\left(a_{i}, r_{i}\right) \neq \emptyset$ for all $i$, then $Y \bigcap\left(\bigcap_{i=1}^{n} B_{X}\left(a_{i}, r_{i}+\varepsilon\right)\right) \neq \emptyset$ for every $(\varepsilon \geq 0) \varepsilon>0$. It is well known that $M$-ideals are precisely the subspaces having the $n$-ball property for all $n \in \mathbb{N}$ (see [7, Chapter I, Theorem 2.2] for details). There is a weaker notion of $M$-ideal called semi $M$-ideal, which is precisely the subspace having the 2-ball property (see [7, Page 43] for details).
In Section 2, we define an almost central subspace of a Banach space by a relative intersection property of balls. We will use this to give some sufficient conditions for subspaces to be central. We also consider some general results about the class (GC). In particular, we prove that an almost central subspace of a Banach space $X$ is in the class (GC) if and only if it is a central subspace of $X^{* *}$. We also derive several sufficient conditions for a semi $M$-ideal to be an $M$-ideal in terms of these intersection properties of balls.

In Section 3, we prove the stability of some of the ball intersection properties in quotient spaces, direct sums, vector-valued continuous function spaces, and injective tensor product spaces (see Chapter VIII of [3] for the theory of injective tensor product spaces). In quotient spaces, we prove that for Banach spaces $X, Y$, and $Z$ with $Z \subseteq Y \subseteq$ $X$, if $Y$ is almost central or ideal in $X$, then $Y / Z$ is almost central or ideal in $X / Z$, respectively, and we also prove the converse when $X$ is an $L_{1}$-predual (that is $X^{*}=L_{1}(\mu)$, for some positive measure $\mu$ ) and $Z$ is an $M$-ideal in $X$. In the case of injective tensor product spaces, we show that if $Z$ is an $L_{1}$-predual space, then, for any almost central subspace $Y$ of a Banach space $X$, the injective tensor product $Z \bigotimes Y$ is an almost central subspace of $Z \&{ }^{\vee} X$. We also prove that properties of being a central subspace and an AC-subspace are stable under a recently introduced concept called polyhedral direct sums of Banach spaces (see [8, Definition 2.1]).

\section{Almost Central Subspaces}

We begin this section with the definition of an "almost central subspace" of a Banach space which is the generalization of the concept central subspace, defined in [2].

Definition 8. A subspace $Y$ of a Banach space $X$ is called an almost central subspace if, for every finite set $\left\{y_{1}, \ldots, y_{n}\right\} \subseteq Y$, $x \in X$, and $\varepsilon>0$, there exists a $y_{\varepsilon} \in Y$ such that $\left\|y_{\varepsilon}-y_{i}\right\| \leq$ $\left\|x-y_{i}\right\|+\varepsilon$ for $1 \leq i \leq n$.

Remark 9. Clearly central subspaces of Banach spaces are almost central. As in the case of central subspace, it is easy to observe that $Y$ is an almost central subspace of a Banach space $X$ if and only if, for each family $\left\{B_{Y}\left(y_{i}, r_{i}\right)\right\}_{i=1}^{n}$ of closed balls in $Y$ having nonempty intersection in $X$, the family $\left\{B_{Y}\left(y_{i}, r_{i}+\right.\right.$ $\varepsilon)\}_{i=1}^{n}$ of closed balls in $Y$ has nonempty intersection in $Y$ for all $\varepsilon>0$. On the other hand, by a weak ${ }^{*}$-compactness argument, it is easy to see that weak ${ }^{*}$-closed almost central subspace of a dual space is a central subspace. Moreover, if $Z$ is an almost central subspace of a Banach space $Y$ and $Y$ is an almost central subspace of a Banach space $X$, then $Z$ is an almost central subspace of $X$.

Lemma 10. Let $X$ be a Banach space and let $Y$ be an ideal in $X$. Then $Y$ is an almost central subspace of $X$.

Proof. Let $\left\{y_{1}, \ldots, y_{n}\right\} \subseteq Y, x \in X$, and $\varepsilon>0$. Choose an $\eta>0$ such that $\eta\left\|x-y_{i}\right\| \leq \varepsilon$ for all $i \in\{1, \ldots, n\}$. 
Define $F=\operatorname{span}\left\{y_{1}, \ldots, y_{n}, x\right\}$. Since $Y$ is an ideal in $X$, by $\left[9\right.$, Theorem 1], there exists an operator $T_{\eta}: F \rightarrow Y$ such that

$$
T_{\eta}(y)=y \quad \text { for } y \in F \cap Y, \quad\left\|T_{\eta}\right\| \leq 1+\eta .
$$

Now define $y_{\eta}=T_{\eta}(x)$. Then $y_{\eta} \in Y$ and for $1 \leq i \leq n$,

$$
\begin{aligned}
\left\|y_{\eta}-y_{i}\right\| & =\left\|T_{\eta}(x)-T_{\eta}\left(y_{i}\right)\right\| \leq(1+\eta)\left\|x-y_{i}\right\| \\
& \leq\left\|x-y_{i}\right\|+\varepsilon .
\end{aligned}
$$

Hence $Y$ is an almost central subspace of $X$.

Since every Banach space is an ideal in its bidual, we have the following result.

Corollary 11. Every Banach space is almost central in its bidual.

Since every $M$-ideal is an ideal, by Lemma $10, M$-ideals are almost central. We now give an example to show that a semi $M$-ideal may not be an almost central subspace.

Example 12. Let $\ell_{1}^{3}$ denote the three-dimensional space $\mathbb{R}^{3}$, endowed with the norm $\|x\|=|x(1)|+|x(2)|+|x(3)|$ for $x=(x(1), x(2), x(3)) \in \mathbb{R}^{3}$. Now consider the subspace $G$ of $\ell_{1}^{3}$ defined as $G=\{(x(1), x(2),-x(1)-x(2)): x(1), x(2) \in$ $\mathbb{R}\} \subseteq \ell_{1}^{3}$. Then, by [7, Chapter I, Remark 2.3(a)], $G$ is a semi $M$-ideal in $\ell_{1}^{3}$. But $G$ is not a central subspace of $\ell_{1}^{3}$. For, let $g_{1}=(-1,-1,2), g_{2}=(-1,2,-1), g_{3}=(2,-1,-1)$, and let $x=(-1,-1,-1)$. Then $g_{1}, g_{2}, g_{3} \in G$ and $x \in \ell_{1}^{3}$. Clearly $\left\|g_{i}-x\right\|=3$ for all $i$. Suppose there is an $\alpha \in G$ such that $\left\|\alpha-g_{i}\right\| \leq 3$ for all $i$. Then

$$
\begin{aligned}
& |\alpha(1)+1|+|\alpha(2)+1|+|\alpha(1)+\alpha(2)+2| \leq 3, \\
& |\alpha(1)+1|+|\alpha(2)-2|+|\alpha(1)+\alpha(2)-1| \leq 3, \\
& |\alpha(1)-2|+|\alpha(2)+1|+|\alpha(1)+\alpha(2)-1| \leq 3 .
\end{aligned}
$$

But (3) shows that both of $\alpha(1)$ and $\alpha$ (2) cannot be positive. But the symmetric inequalities (4) and (5) rule out other possibilities. Thus $G$ is not a central subspace of $\ell_{1}^{3}$. Then, by a compactness argument, we can see that $G$ is not an almost central subspace of $\ell_{1}^{3}$.

In [1, Example 5.6], Veselý gave an example of a threedimensional Banach space $X$ such that $C([0,1], X)$ is not a central subspace of its bidual. Since every Banach space is an ideal in its bidual, the same example shows that an ideal (in particular, an almost central subspace) need not be a central subspace. We now give a sufficient condition for an almost central subspace to be a central subspace.

Theorem 13. Let $Y$ be an almost central subspace of a Banach space $X$ such that $Y \in(G C)$. Then $Y$ is a central subspace of $X$.

Proof. Let $\left\{y_{1}, \ldots, y_{n}\right\} \subseteq Y$ and $x \in X$. Since $Y \in$ (GC), by [2, Proposition 2.9], it is enough to show that $\bigcap_{i=1}^{n} B_{Y}\left(y_{i}, \| x-\right.$ $\left.y_{i} \|+\varepsilon\right) \neq \emptyset$ for all $\varepsilon>0$.
Now let $\varepsilon>0$. Choose $\eta>0$ such that $\eta\left\|x-y_{i}\right\| \leq \varepsilon$ for all $i \in\{1, \ldots, n\}$. Since $Y$ is an almost central subspace of $X$, there exists a $y \in Y$ such that $\left\|y-y_{i}\right\| \leq(1+\eta)\left\|x-y_{i}\right\|$ for all $i \in\{1, \ldots, n\}$. Hence $\left\|y-y_{i}\right\| \leq\left\|x-y_{i}\right\|+\varepsilon$ for all $i \in\{1, \ldots, n\}$, and the result follows.

Our next result gives a sufficient condition for an almost central subspace to be an AC-subspace.

Proposition 14. Let $Y$ be an almost central subspace of a Banach space $X$ such that $Y$ is isometric to the range of a projection of norm one in some dual space. Then $Y$ is an ACsubspace of $X$.

Proof. Let $Z$ be a Banach space and $P: Z^{*} \rightarrow Z^{*}$ be a projection of norm one such that $Y$ is isometric to range $(P)$. Let $\phi: Y \rightarrow \operatorname{range}(P)$ be the corresponding onto isometry. Now let $\left\{B_{Y}\left(y_{\alpha}, r_{\alpha}\right)\right\}_{\alpha \in I}$ be any family of closed balls in $Y$ and $x \in X$ be such that $\left\|x-y_{\alpha}\right\| \leq r_{\alpha}$ for all $\alpha \in I$. Consider the family $\left\{B_{Y}\left(y_{\alpha}, r_{\alpha}+\varepsilon\right)\right\}_{\alpha \in I, \varepsilon>0}$. Since $Y$ is an almost central subspace of $X$, any finite collection of balls from this family has nonempty intersection in $Y$. Hence any finite collection of balls from the family $\left\{B_{Z^{*}}\left(\phi\left(y_{\alpha}\right), r_{\alpha}+\varepsilon\right)\right\}_{\alpha \in I, \mathcal{E}>0}$ has nonempty intersection in $\operatorname{range}(P)$. Now, by weak ${ }^{*}$ compactness, there exists a $\Lambda \in Z^{*}$ such that $\left\|\Lambda-\phi\left(y_{\alpha}\right)\right\| \leq r_{\alpha}+\varepsilon$ for all $\alpha \in I$ and for all $\varepsilon>0$. Hence $\left\|\Lambda-\phi\left(y_{\alpha}\right)\right\| \leq r_{\alpha}$ for all $\alpha \in I$. Now define $y=\phi^{-1}(P(\Lambda))$. Then, for all $\alpha \in I$, we have

$$
\begin{aligned}
\left\|y-y_{\alpha}\right\| & =\left\|\phi^{-1}(P(\Lambda))-\phi^{-1}\left(\phi\left(y_{\alpha}\right)\right)\right\| \\
& =\left\|P(\Lambda)-\phi\left(y_{\alpha}\right)\right\| \\
& =\left\|P\left(\Lambda-\phi\left(y_{\alpha}\right)\right)\right\| \\
& \leq r_{\alpha} .
\end{aligned}
$$

Hence $Y$ is an AC-subspace of $X$.

We now give a class of Banach spaces where almost central subspaces are central. We recall that a Banach space $X$ whose dual $X^{*}$ is isometric to $L_{1}(\mu)$ for some positive measure $\mu$ is called an $L_{1}$-predual.

Proposition 15. Let $X$ be an $L_{1}$-predual and let $Y$ be an almost central subspace of $X$. Then $Y$ is an $L_{1}$-predual. In particular, $Y$ is a central subspace of $X$.

Proof. Let $\left\{B_{Y}\left(y_{i}, r_{i}\right)\right\}_{i=1}^{n}$ be any family of $n$ balls in $Y$ such that any two of them intersect in $Y$. Since $X$ is an $L_{1}$-predual, by [10, Theorem 6.1], there exists an $x \in X$ such that $\left\|x-y_{i}\right\| \leq r_{i}$ for all $i$. Also, since $Y$ is an almost central subspace of $X$, $\bigcap_{i=1}^{n} B_{Y}\left(y_{i}, r_{i}+\varepsilon\right) \neq \emptyset$ for all $\varepsilon>0$. Then, by [10, Lemma 4.2 and Theorem 6.1], it follows that $Y$ is an $L_{1}$-predual. Now let $\left\{B_{Y}\left(y_{i}, r_{i}\right)\right\}_{i=1}^{n}$ be a family of $n$ balls in $Y$ that has nonempty intersection in $X$. It is well known that two balls intersect if and only if the distance between the centers is less than or equal to the sum of the radii. Thus $\left\{B_{Y}\left(y_{i}, r_{i}\right)\right\}_{i=1}^{n}$ is a pairwise intersecting family in $Y$. Since $Y$ is an $L_{1}$-predual, by [10, Theorem 6.1], it follows that $\left\{B_{Y}\left(y_{i}, r_{i}\right)\right\}_{i=1}^{n}$ intersects in $Y$. Hence $Y$ is a central subspace of $X$. 
Remark 16. Following the same line of argument as in the proof of [2, Theorem 3.3], we can observe that a Banach space $X$ is an $L_{1}$-predual if and only if $X$ is an almost central subspace of every Banach space that contains it.

Since every ideal is almost central, our next result generalizes Proposition 14 of [11].

Proposition 17. Let $Y$ be an almost central subspace of a Banach space $X$. Then $Y$ is a central subspace of $X^{* *}$ if and only if $Y \in(G C)$.

Proof. If $Y$ is a central subspace of $X^{* *}$, then, by [2, Proposition 2.2(d)], $Y \in(\mathrm{GC})$.

Conversely suppose $Y \in(\mathrm{GC})$. Since $Y$ is an almost central subspace of $X$ and $X$ is an almost central subspace of $X^{* *}$, by Remark $9, Y$ is an almost central subspace of $X^{* *}$. Hence, by Theorem 13, it follows that $Y$ is a central subspace of $X^{* *}$.

By a similar transitivity argument used in the proof of the Proposition 17, we can easily observe the following corollaries.

Corollary 18. Let $Y$ be a subspace of $X$ such that $Y^{\perp \perp}$ is an almost central subspace of $X^{* *}$. Then $Y$ is an almost central subspace of $X^{* *}$. In addition, if $Y \in(G C)$, then $Y$ is a central subspace of $X^{* *}$.

It is well known that a semi $M$-ideal need not be an $M$ ideal (see [7, Chapter I, Remarks 2.3(a)] for example). Our next theorem gives a sufficient condition for a semi $M$-ideal to be an $M$-ideal.

Theorem 19. Let $Y$ be a semi $M$-ideal in a Banach space $X$ such that $Y^{\perp \perp}$ is an almost central subspace of $X^{* *}$. Then $Y$ is an $M$-ideal in $X$.

Proof. Since $Y$ is a semi $M$-ideal, by [12, Theorem 6.14], $Y^{\perp \perp}$ is a semi $M$-ideal in $X^{* *}$. Also, since $Y^{\perp \perp}$ is a weak ${ }^{*}$-closed almost central subspace of $X^{* *}, Y^{\perp \perp}$ is an AC-subspace of $X^{* *}$. Hence, for any $\Lambda \notin Y^{\perp \perp}$, by [4, Proposition 2.2], $Y^{\perp \perp}$ is 1-complemented in $\operatorname{span}\left\{Y^{\perp \perp}, \Lambda\right\}$ and hence is an ideal in $\operatorname{span}\left\{Y^{\perp \perp}, \Lambda\right\}$. Now, for any $\Lambda \notin Y^{\perp \perp}$, since $Y^{\perp \perp}$ is a semi $M$ ideal in $\operatorname{span}\left\{Y^{\perp \perp}, \Lambda\right\}$, by [11, Proposition 23], it follows that $Y^{\perp \perp}$ is an $M$-ideal in $\operatorname{span}\left\{Y^{\perp \perp}, \Lambda\right\}$. Hence, by [7, Chapter I, Theorem 2.2], $Y^{\perp \perp}$ is an $M$-ideal in $X^{* *}$. Since $Y^{\perp \perp}$ is a weak $^{*}$-closed $M$-ideal in $X^{* *}$, by [7, Chapter II, Corollary 3.6], $Y^{\perp \perp}$ is an $M$-summand in $X^{* *}$. Hence, by [7, Chapter I, Theorem 1.9], there exists an $L$-summand $V$ in $X^{*}$ such that $X^{* *}=Y^{\perp \perp} \bigoplus_{\infty} V^{\perp}$. Then, by the duality between $L$ - and $M$ projections, we get $X^{*}=Y^{\perp} \bigoplus_{1} V$ and hence $Y$ is an $M$-ideal in $X$.

We now give a sufficient condition for a semi $M$-ideal to be an $M$-summand.

Theorem 20. Let $Y$ be an AC-subspace of a Banach space $X$. Then $Y$ is a semi $M$-ideal in $X$ if and only if $Y$ is an $M$ summand in $X$.
Proof. Suppose $Y$ is a semi $M$-ideal in $X$ and is an ACsubspace of $X$. Since $Y$ is an AC-subspace of $X$, by [4, Proposition 2.2], $Y$ is 1-complemented in $\operatorname{span}\{Y, x\}$ for all $x \in X$. Also, since $Y$ is a semi $M$-ideal in $X, Y$ is a semi $M$ ideal in $\operatorname{span}\{Y, x\}$ for all $x \in X$. Thus, by [11, Proposition 23], $Y$ is an $M$-ideal in $\operatorname{span}\{Y, x\}$ for all $x \in X$. Then, by [7, Chapter I, Corollary 1.3], $Y$ is an $M$-summand in $\operatorname{span}\{Y, x\}$ for all $x \in X$. Hence, by [7, Chapter II, Proposition 3.2], $Y$ is an $M$-summand in $X$.

Our next theorem gives another sufficient condition for a semi $M$-ideal to be an $M$-ideal. In fact, this result improves Proposition 23 of [11].

Theorem 21. Let $Y$ be a subspace of a Banach space $X$ such that $Y$ is an ideal in $\operatorname{span}\{Y, x\}$ for all $x \in X$. Then $Y$ is a semi $M$-ideal in $X$ if and only if $Y$ is an $M$-ideal in $X$.

Proof. Suppose $Y$ is a semi $M$-ideal in $X$ and is an ideal in $\operatorname{span}\{Y, x\}$ for all $x \in X$. Then, by [11, Proposition 23], $Y$ is an $M$-ideal in $\operatorname{span}\{Y, x\}$ for all $x \in X$. Hence, by [7, Chapter I, Theorem 2.2], it follows that $Y$ is an $M$-ideal in $X$.

We now recall the following theorem of Bandyopadhyay and Dutta that characterizes an AC-subspace of finite codimension in the space $C(K)$ of all continuous real-valued functions on a compact Hausdorff space $K$, endowed with the supremum norm.

Theorem 22 (see [5, Theorem 1.1]). Let $K$ be a compact Hausdorff space and $Y$ be a subspace of codimension n of $C(K)$. Then the following are equivalent.

(i) $Y$ is an AC-subspace of $C(K)$.

(ii) $Y$ is 1-complemented in $C(K)$.

(iii) There exist measures $\mu_{1}, \ldots, \mu_{n}$ on $K$ and distinct isolated points $k_{1}, \ldots, k_{n}$ of $K$ such that
(a) $Y=\bigcap_{i=1}^{n} \operatorname{ker}\left(\mu_{i}\right)$
(b) $\left\|\mu_{i}\right\| \leq 2\left|\mu_{i}\left(\left\{k_{i}\right\}\right)\right|, i=1, \ldots, n$.

In our next proposition, we observe a simple proof for the implication (iii) $\Rightarrow$ (ii) of Theorem 22 when $K$ is an extremely disconnected space.

We recall that a compact Hausdorff space $K$ is extremely disconnected if the closure of each open set in $K$ is again open in $K$ (see [13, Section 7] for details).

For any infinite discrete set $\Gamma, \ell_{\infty}(\Gamma)$ denotes the space of all bounded real-valued functions on $\Gamma$, endowed with the supremum norm, and $c_{0}(\Gamma)$ denotes its subspace consisting of all functions $f \in \ell_{\infty}(\Gamma)$ such that the set $\{\gamma \in \Gamma:|f(\gamma)| \geq$ $\varepsilon\}$ is finite for all $\varepsilon>0$. Also, for any infinite discrete set $\Gamma$, $\ell_{1}(\Gamma)$ denotes the space of all functions $f: \Gamma \rightarrow \mathbb{R}$ such that $\sum_{\gamma \in \Gamma}|f(\gamma)|<\infty$ with the norm $\|f\|_{1}=\sum_{\gamma \in \Gamma}|f(\gamma)|$.

The following lemma is the uncountable version of the main theorem of [14] for the space $\ell_{\infty}(\Gamma)$. As the proof is similar to that of the theorem of [14], we omit the proof here. 
Lemma 23. Let $\Gamma$ be any infinite discrete set and $Y$ be a subspace of codimension $n$ in $\ell_{\infty}(\Gamma)$. Then $Y$ is 1-complemented in $\ell_{\infty}(\Gamma)$ if and only if there exist $n$ distinct elements $t_{1}, \ldots, t_{n}$ in $\Gamma$ and $n$ linearly independent functionals $f_{1}, \ldots, f_{n}$ in $\left(\ell_{\infty}(\Gamma)\right)^{*}$ such that

(a) $f_{i}=h_{i}+g_{i}$ with $h_{i} \in l_{1}(\Gamma), g_{i} \in c_{0}(\Gamma)^{\perp}, i=1, \ldots, n$,

(b) $Y=\bigcap_{i=1}^{n} f_{i}^{-1}(0)$,

(c) $\left\|g_{i}\right\| \leq 2\left|h_{i}\left(t_{i}\right)\right|-\left\|h_{i}\right\|_{1}, i=1, \ldots, n$.

Our next result shows that the space $\ell_{\infty}(\Gamma)$ cannot have a finite codimensional 1-complemented subspace containing $c_{0}(\Gamma)$.

Corollary 24. Let $X$ be a Banach space such that $c_{0}(\Gamma) \subset$ $X \subset \ell_{\infty}(\Gamma)$ for some infinite discrete space $\Gamma$. If $X$ is a finite codimensional subspace of $\ell_{\infty}(\Gamma)$, then $X$ cannot be 1 complemented in $\ell_{\infty}(\Gamma)$.

Proof. Suppose $X$ is 1-complemented in $\ell_{\infty}(\Gamma)$. Then, by Lemma 23, there exist $n$ distinct elements $t_{1}, \ldots, t_{n}$ in $\Gamma$ and $n$ linearly independent functionals $f_{1}, \ldots, f_{n}$ in $\left(\ell_{\infty}(\Gamma)\right)^{*}$ such that

(a) $f_{i}=h_{i}+g_{i}$ with $h_{i} \in l_{1}(\Gamma), g_{i} \in c_{0}(\Gamma)^{\perp}, i=1, \ldots, n$,

(b) $X=\bigcap_{i=1}^{n} f_{i}^{-1}(0)$,

(c) $\left\|g_{i}\right\| \leq 2\left|h_{i}\left(t_{i}\right)\right|-\left\|h_{i}\right\|_{1}, i=1, \ldots, n$.

Since $X^{\perp} \subseteq c_{0}(\Gamma)^{\perp}, f_{i} \in c_{0}(\Gamma)^{\perp}$ for all $i \in\{1, \ldots, n\}$. Hence $h_{i}=f_{i}-g_{i} \in c_{0}(\Gamma)^{\perp}$ for all $i \in\{1, \ldots, n\}$. Since $\ell_{\infty}(\Gamma)^{*}=$ $\ell_{1}(\Gamma) \bigoplus_{1} c_{0}(\Gamma)^{\perp}$, we get $h_{i}=0$ for all $i \in\{1, \ldots, n\}$. Then, by (c), $g_{i}=0$ and hence $f_{i}=0$ for all $i \in\{1, \ldots, n\}$. This contradiction proves that $X$ cannot be 1-complemented in $\ell_{\infty}(\Gamma)$.

Let $K$ be a compact Hausdorff space and $E$ be a closed subset of $K$. Also, let $\mathscr{B}(K)$ be the class of Borel subsets of $K$. Now, for $\mu \in C(E)^{*}$, we define $\widetilde{\mu} \in C(K)^{*}$ as

$$
\widetilde{\mu}(B)= \begin{cases}\mu(B), & \text { if } B \in \mathscr{B}(K), B \subset E, \\ 0, & \text { if } B \in \mathscr{B}(K), B \bigcap E=\emptyset .\end{cases}
$$

Lemma 25. Let $K$ be a compact Hausdorff space and let $E$ be a closed subset of $K$ such that there exists a continuous map $\varphi$ : $K \rightarrow E$ which is identity on $E$ and let $\mu_{i} \in C(E)^{*}(1 \leq i \leq n)$. If $\bigcap_{i=1}^{n} \operatorname{ker}\left(\widetilde{\mu_{i}}\right)$ is 1-complemented in $C(K)$, then $\bigcap_{i=1}^{n} \operatorname{ker}\left(\mu_{i}\right)$ is 1 -complemented in $C(E)$.

Proof. Let $P: C(K) \rightarrow C(K)$ be a projection of norm one with range $\bigcap_{i=1}^{n} \operatorname{ker}\left(\widetilde{\mu_{i}}\right)$.

Now define $P^{\prime}: C(E) \rightarrow C(E)$ by

$$
P^{\prime}(f)=\left.P(f \circ \varphi)\right|_{E} \quad \text { for } f \in C(E) .
$$

Since

$$
\begin{aligned}
\int_{E} P^{\prime}(f) d \mu_{i} & =\left.\int_{E} P(f \circ \varphi)\right|_{E} d \mu_{i}=\int_{K} P(f \circ \varphi) d \widetilde{\mu_{i}} \\
& =0 \quad \forall f \in C(E),
\end{aligned}
$$

we get $P^{\prime}(f) \in \bigcap_{i=1}^{n} \operatorname{ker}\left(\mu_{i}\right)$ and hence $P^{\prime}$ is well defined. Clearly $P^{\prime}$ is a linear map.

Now let $f \in \bigcap_{i=1}^{n} \operatorname{ker}\left(\mu_{i}\right)$. Since $\varphi$ is identity on $E$, we have

$$
\int_{K} P(f \circ \varphi) d \widetilde{\mu_{i}}=\int_{E} f d \mu_{i}=0 \quad \forall i .
$$

Thus, $f \circ \varphi \in \bigcap_{i=1}^{n} \operatorname{ker}\left(\widetilde{\mu_{i}}\right)$ and $P(f \circ \varphi)=f \circ \varphi$. Therefore $P^{\prime}(f)=\left.f \circ \varphi\right|_{E}=f$ and hence $P^{\prime}$ is a projection on $C(E)$ with range $\bigcap_{i=1}^{n} \operatorname{ker}\left(\mu_{i}\right)$. Now, since $\left\|P^{\prime}(f)\right\|=\left\|\left.P(f \circ \varphi)\right|_{E}\right\| \leq$ $\|P(f \circ \varphi)\| \leq\|f \circ \varphi\|=\|f\|,\left\|P^{\prime}\right\|=1$. Hence $P^{\prime}$ is the required projection.

Proposition 26. Let $K$ be an extremely disconnected space. If there exist measures $\mu_{1}, \ldots, \mu_{n}$ on $K$ and distinct isolated points $k_{1}, \ldots, k_{n}$ of $K$ such that $\left\|\mu_{i}\right\| \leq 2\left|\mu_{i}\left(\left\{k_{i}\right\}\right)\right|$ for $1 \leq i \leq n$, then $\bigcap_{i=1}^{n} \operatorname{ker}\left(\mu_{i}\right)$ is 1-complemented in $C(K)$.

Proof. Let $\Gamma$ be a dense subset of $K$. Since each $k_{i}$ 's are isolated points of $K, k_{i} \in \Gamma$ for all $i$. Now consider $\Gamma$ with the discrete topology and its Stone-Čech compactification $\beta(\Gamma)$. Then, by [13, Section 7, Lemma 3 and Theorem 3], $K$ is homeomorphically embedded into $\beta(\Gamma)$, and also there exists a continuous map $\varphi: \beta(\Gamma) \rightarrow K$ such that $\varphi$ is identity on $K$. Now consider measures $\widetilde{\mu_{i}}$ on $\beta(\Gamma)$ such that $\widetilde{\mu_{i}}(D)=0$ for any Borel set $D$ disjoint from $K$ and $\widetilde{\mu}_{i}(D)=\mu_{i}(D)$ for any Borel set $D \subset K$. Since $k_{i} \in \Gamma, 2\left|\mu_{i}\left(\left\{k_{i}\right\}\right)\right| \geq\left\|\mu_{i}\right\|=\|\widetilde{\mu}\|$. Since $C(\beta(\Gamma))$ is isometric to $\ell_{\infty}(\Gamma)$, by Lemma $23, \bigcap_{i=1}^{n} \operatorname{ker}\left(\widetilde{\mu_{i}}\right)$ is 1 -complemented in $\ell_{\infty}(\Gamma)$. Then, by Lemma $25, \bigcap_{i=1}^{n} \operatorname{ker}\left(\mu_{i}\right)$ is 1-complemented in $C(K)$.

In an $L_{1}$-predual space, we do not know whether every AC-subspace of finite codimension is the range of a norm one projection and/or is the intersection of AC-subspaces of codimension one.

\section{Stability Results}

Coming to quotient spaces, one can easily observe that if $Y$ is 1-complemented in a Banach space $X$, then, for any subspace $Z$ of $Y, Y / Z$ is 1 -complemented in $X / Z$. Motivated by this, we consider the following problem. Let $Y$ be a subspace of a Banach space $X$ having some property $(P)$ in $X$. If $Z$ is a subspace of $Y$, then when can we say that $Y / Z$ has the property $(P)$ in $X / Z$ ? We study this problem when the property $(P)$ under consideration is almost constrained, almost central, central, and ideal.

For a subspace $Y$ of a Banach space $X$ and $x \in X$, we denote by $[x]$ the equivalence class in $X / Y$ containing $x$.

Our next result solves the above problem for AC-subspaces.

Proposition 27. Let $Y$ be an $A C$-subspace of $X$ and let $Z$ be a subspace of $Y$. Then $Y / Z$ is an AC-subspace of $X / Z$. 
Proof. Let $\left\{B_{Y / Z}\left(\left[y_{i}\right], r_{i}\right)\right\}_{i \in I}$ be a family of balls in $Y / Z$ and also let $x \in X$ be such that $[x] \in \bigcap_{i \in I} B_{Y / Z}\left(\left[y_{i}\right], r_{i}\right)$. Then, for each $\varepsilon>0$ and $i \in I$, there exists a $z_{\varepsilon, i} \in Z$ such that

$$
\left\|x-y_{i}+z_{\varepsilon, i}\right\| \leq\left\|[x]-\left[y_{i}\right]\right\|+\varepsilon \leq r_{i}+\varepsilon \quad \forall i \in I, \varepsilon>0 .
$$

We now consider the family $\left\{B_{Y}\left(y_{i}-z_{\varepsilon, i}, r_{i}+\varepsilon\right)\right\}_{i \in I, \varepsilon>0}$ of closed balls in $Y$. Clearly $x \in \bigcap_{i \in I, \varepsilon>0} B_{Y}\left(y_{i}-z_{\varepsilon, i}, r_{i}+\varepsilon\right)$. Since $Y$ is an AC-subspace of $X$, there exists a $y \in Y$ such that $y \in$ $\bigcap_{i \in I, \varepsilon>0} B_{Y}\left(y_{i}-z_{\varepsilon, i}, r_{i}+\varepsilon\right)$. Then, for $i \in I$, we have

$$
\left\|[y]-\left[y_{i}\right]\right\| \leq\left\|y-y_{i}+z_{\varepsilon, i}\right\| \leq r_{i}+\varepsilon \quad \forall \varepsilon>0 .
$$

Therefore $\left\|[y]-\left[y_{i}\right]\right\| \leq r_{i}$ for all $i \in I$, and hence $Y / Z$ is an AC-subspace of $X / Z$.

We now prove the stability of ideals in quotient spaces.

Proposition 28. Let $Y$ be an ideal in $X$ and let $Z$ be a subspace of $Y$. Then $Y / Z$ is an ideal in $X / Z$.

Proof. Since $Y$ is an ideal in $X$, by [9, Theorem 1], $Y^{\perp \perp}$ is 1 complemented in $X^{* *}$. Then $Y^{\perp \perp} / Z^{\perp \perp}$ is 1 -complemented in $X^{* *} / Z^{\perp \perp}$. But $X^{* *} / Z^{\perp \perp}$ is isometric to $(X / Z)^{* *}$, and this isometry takes $Y^{\perp \perp} / Z^{\perp \perp}$ onto $(Y / Z)^{\perp \perp}$. Hence $(Y / Z)^{\perp \perp}$ is 1 complemented in $(X / Z)^{* *}$. Then, again by [9, Theorem 1], $Y / Z$ is an ideal in $X / Z$.

Our next result proves the stability of almost central subspaces in quotient spaces.

Proposition 29. Let $Y$ be an almost central subspace of $X$ and let $Z$ be a subspace of $Y$. Then $Y / Z$ is an almost central subspace of $X / Z$.

Proof. Let $[x] \in X / Z,\left\{\left[y_{1}\right], \ldots,\left[y_{n}\right]\right\} \subseteq Y / Z$ and $\varepsilon>0$. Then, for $1 \leq i \leq n$, there exists an element $z_{\varepsilon, i} \in Z$ such that

$$
\left\|x-y_{i}+z_{\varepsilon, i}\right\| \leq\left\|[x]-\left[y_{i}\right]\right\|+\frac{\varepsilon}{2} .
$$

Since $Y$ is an almost central subspace of $X$, there exists an element $y_{\varepsilon} \in Y$ such that

$$
\left\|y_{\varepsilon}-y_{i}+z_{\varepsilon, i}\right\| \leq\left\|x-y_{i}+z_{\varepsilon, i}\right\|+\frac{\varepsilon}{2} \quad \text { for } 1 \leq i \leq n
$$

Now, for $1 \leq i \leq n$, we have

$$
\begin{aligned}
\left\|\left[y_{\varepsilon}\right]-\left[y_{i}\right]\right\| & \leq\left\|y_{\varepsilon}-y_{i}+z_{\varepsilon, i}\right\| \leq\left\|x-y_{i}+z_{\varepsilon, i}\right\|+\frac{\varepsilon}{2} \\
& \leq\left\|[x]-\left[y_{i}\right]\right\|+\varepsilon .
\end{aligned}
$$

Hence $Y / Z$ is an almost central subspace of $X / Z$.

Now, for Banach spaces $X, Y, Z$ with $Z \subseteq Y \subseteq X$, our next set of results give some sufficient conditions for $Y / Z$ to be a central subspace of $X / Z$.

Combining Proposition 29 and Theorem 13, we get the following.
Corollary 30. Let $Y$ be an almost central subspace of $X$ and let $Z$ be a subspace of $Y$. If $Y / Z \in(G C)$, then $Y / Z$ is a central subspace of $X / Z$.

As a consequence of the above corollary, we have the following result.

Corollary 31. Let $Y$ be a subspace of $X$ and let $Z$ be a subspace of $Y$ such that $Y / Z \in(G C)$. If $Y^{\perp \perp}$ is an almost central subspace of $X^{* *}$, then $Y / Z$ is a central subspace of $X / Z$.

Proof. By Corollary 18, $Y$ is an almost central subspace of $X^{* *}$. Hence $Y$ is an almost central subspace of $X$. Since $Y / Z \in$ (GC), by Corollary $30, Y / Z$ is a central subspace of $X / Z$.

We recall that a subspace $Y$ of a Banach space $X$ is said to be a factor reflexive subspace if the quotient space $X / Y$ is reflexive. Since any reflexive spaces are in the class (GC), the following corollary is easy to see.

Corollary 32. Let $Y$ be a subspace of a Banach space $X$ such that $Y^{\perp \perp}$ is an almost central subspace of $X^{* *}$. Then, for any factor reflexive subspace $Z$ of $Y, Y / Z$ is a central subspace of $X / Z$.

We now prove the converse of Proposition 29 under some additional assumptions.

Proposition 33. Let $X$ be an $L_{1}$-predual, let $Z$ be an $M$-ideal in $X$, and let $Y$ be a subspace of $X$ such that $Z \subseteq Y \subseteq X$. If $Y / Z$ is almost central in $X / Z$, then $Y$ is a central subspace of $X$.

Proof. Let $x \in X, y_{1} \ldots, y_{n} \in Y$, and $\varepsilon>0$. Then, by assumption, there exists a $y_{\varepsilon} \in Y$ such that

$$
\left\|\left[y_{\varepsilon}\right]-\left[y_{i}\right]\right\| \leq\left\|[x]-\left[y_{i}\right]\right\|+\frac{\varepsilon}{2} \leq\left\|x-y_{i}\right\|+\frac{\varepsilon}{2} .
$$

Let $z_{\varepsilon, i} \in Z$ be such that $\left\|y_{\varepsilon}-y_{i}-z_{i}\right\| \leq\left\|x-y_{i}\right\|+\varepsilon$ for all $i \in\{1, \ldots, n\}$. Now consider the finite collection of balls $\left\{B_{X}\left(y_{\varepsilon}-y_{i},\left\|x-y_{i}\right\|+\varepsilon\right)\right\}_{i=1}^{n}$ in $X$. Since this is a pairwise intersecting family of balls in $X$ and $X$ is an $L_{1}$-predual, $\bigcap_{i=1}^{n} B_{X}\left(y_{\varepsilon}-y_{i},\left\|x-y_{i}\right\|+\varepsilon\right) \neq \emptyset$. Also, since $X$ is an $L_{1}$-predual, by [12, Proposition 6.5], it follows that $Z$ has the strong $n$-ball property in $X$. Hence there exists an element $z_{\varepsilon} \in Z$ such that $\left\|z_{\varepsilon}-y_{\varepsilon}+y_{i}\right\| \leq\left\|x-y_{i}\right\|+\varepsilon$ for all $i \in\{1, \ldots, n\}$. Therefore $Y$ is an almost central subspace of $X$ and hence, by Proposition 15, $Y$ is a central subspace of $X$.

The following corollary is the converse of Proposition 28 under some additional assumptions.

Corollary 34. Let $X$ be an $L_{1}$-predual, let $Z$ be an $M$-ideal in $X$, and let $Y$ be a subspace of $X$ such that $Z \subseteq Y \subseteq X$. If $Y / Z$ is an ideal in $X / Z$, then $Y$ is an ideal in $X$.

Proof. Since $Y / Z$ is an ideal in $X / Z$, by Lemma $10, Y / Z$ is almost central in $X / Z$. Thus, by Proposition 33, $Y$ is a central subspace of $X$. Then, by Proposition $15, Y$ is an $L_{1}$-predual. Hence, by [15, Proposition 1], $Y$ is an ideal in $X$. 
We recall that, for any collection $\left\{X_{\alpha}: \alpha \in \Gamma\right\}$ of Banach spaces, $\ell_{\infty}$-sum of $X_{\alpha}(\alpha \in \Gamma)$ and $c_{0}$-sum of $X_{\alpha}(\alpha \in \Gamma)$ are defined as $\bigoplus_{\infty} X_{\alpha}=\left\{x \in \prod X_{\alpha}:\|x\|=\sup \|x(\alpha)\|<\infty\right\}$ and $\bigoplus_{c_{0}} X_{\alpha}=\left\{x \in \prod X_{\alpha}\right.$ : for all $\varepsilon>0,\{\alpha \in \Gamma:\|x(\alpha)\|>$ $\varepsilon\}$ is finite $\}$, respectively, and equip both spaces with supremum norm. For a finite family of Banach spaces $\left\{X_{1}, \ldots, X_{k}\right\}$, $\ell_{\infty}$-sum of $X_{i}(1 \leq i \leq k)$ is denoted by $\left(X_{1} \bigoplus \cdots \bigoplus X_{k}\right)_{\ell_{\infty}^{k}}$.

Remark 35. It is easy to observe that, for any family $\left\{X_{\alpha}: \alpha \epsilon\right.$ $\Gamma\}$ of Banach spaces, if $Y_{\alpha}$ is an almost central subspace of a Banach space $X_{\alpha}$, then $\bigoplus_{\infty} Y_{\alpha}$ is an almost central subspace of $\bigoplus_{\infty} X_{\alpha}$.

We now prove the stability of almost central subspaces in vector-valued continuous function spaces. For a compact Hausdorff space $K$ and a Banach space $X$, we denote by $C(K, X)$ the space of all $X$-valued continuous functions defined on $K$, endowed with the supremum norm.

Let $K$ be a compact Hausdorff space and let $X$ be a Banach space. Then, for $f \in C(K)$ and $x \in X$, an element $f \otimes x \in$ $C(K, X)$ is defined as $(f \otimes x)(k)=f(k) x$ for $k \in K$.

Proposition 36. Let $Y$ be an almost central subspace of Banach space $X$ and let $K$ be a compact Hausdorff space. Then $C(K, Y)$ is an almost central subspace of $C(K, X)$.

Proof. Let $f_{1}, \ldots, f_{n} \in C(K, Y), f \in C(K, X)$, and $\varepsilon>0$. Then, by the proof of [10, Page 43, Corollary 2], for the finite family $\left\{f_{1}, \ldots, f_{n}\right\}$, there exists a partition of unity $\left\{\varphi_{j}\right\}_{j=1}^{m}$ and a closed subspace $B$ of $C(K, Y)$ spanned by the elements of the form $\sum_{j=1}^{m} \varphi_{j} \otimes y_{j}$ with $y_{j} \in Y$ such that $d\left(f_{i}, B\right)<\varepsilon / 4$ for $1 \leq$ $i \leq n$ and $B$ is isometric to $(Y \oplus \cdots \bigoplus Y)_{\ell_{\infty}^{m}}$. Similarly for $f$, there exists a partition of unity $\left\{\varphi_{l}^{\prime}\right\}_{l=1}^{k}$ and a closed subspace $B^{\prime}$ of $C(K, X)$ spanned by the elements of the form $\sum_{l=1}^{k} \varphi_{l}^{\prime} \otimes x_{l}$ with $x_{l} \in X$ such that $d\left(f, B^{\prime}\right)<\varepsilon / 4$, and $B^{\prime}$ is isometric to $(X \bigoplus \cdots \oplus X)_{\ell_{\infty}^{k}}$. Now let $\tilde{f} \in B^{\prime}$ be such that $\|f-\tilde{f}\|<\varepsilon / 4$ and let $\widetilde{f}_{i} \in B$ be such that $\left\|f_{i}-\widetilde{f}_{i}\right\|<\varepsilon / 4$ for $1 \leq i \leq n$.

Case $1(m \leq k)$. Since $B$ is isometric to $(Y \oplus \cdots \bigoplus Y)_{\ell_{\infty}^{m}}, B$ is an $M$-summand in $(Y \oplus \cdots \bigoplus Y)_{\ell_{\infty}^{k}}$ (up to an isometry). Since $M$-summands are central, by Remark 35 and Remark 9 , $B$ is an almost central subspace of $B^{\prime}$. Then there exists a $g \in B$ such that $\left\|g-\widetilde{f}_{i}\right\| \leq\left\|\tilde{f}-\widetilde{f}_{i}\right\|+\varepsilon / 4$ for $1 \leq i \leq n$. Hence we have

$$
\begin{aligned}
\left\|g-f_{i}\right\| & \leq\left\|g-\widetilde{f}_{i}\right\|+\left\|\widetilde{f}_{i}-f_{i}\right\| \\
& \leq\left\|\tilde{f}-\widetilde{f}_{i}\right\|+\frac{\varepsilon}{4}+\frac{\varepsilon}{4} \\
& \leq\|\tilde{f}-f\|+\left\|f-f_{i}\right\|+\left\|f_{i}-\widetilde{f}_{i}\right\|+\frac{\varepsilon}{2} \\
& \leq\left\|f-f_{i}\right\|+\varepsilon .
\end{aligned}
$$

Case $2(k<m)$. In this case, we can isometrically embed $B^{\prime}$ into $(X \bigoplus \cdots \oplus X)_{\ell_{\infty}^{m}}$. Since $B$ is isometric to $(Y \oplus \cdots$ $\bigoplus Y)_{\ell_{\infty}^{m}}$, by Remark 35, $B$ is an almost central subspace of
$(X \bigoplus \cdots \oplus X)_{\ell_{\infty}^{m}}$ (up to an isometry). Then there exists an element $g \in B$ such that $\left\|g-\widetilde{f}_{i}\right\| \leq\left\|\widetilde{f}-\widetilde{f}_{i}\right\|+\varepsilon / 4$ for $1 \leq i \leq n$. Hence we have

$$
\begin{aligned}
\left\|g-f_{i}\right\| & \leq\left\|g-\widetilde{f}_{i}\right\|+\left\|\widetilde{f}_{i}-f_{i}\right\| \\
& \leq\|\tilde{f}-f\|+\left\|f-f_{i}\right\|+\left\|f_{i}-\widetilde{f}_{i}\right\|+\frac{\varepsilon}{2} \\
& \leq\left\|f-f_{i}\right\|+\varepsilon .
\end{aligned}
$$

Therefore in all cases there exists a $g \in B \subseteq C(K, Y)$ such that $\left\|g-f_{i}\right\| \leq\left\|f-f_{i}\right\|+\varepsilon$ for $1 \leq i \leq n$. Hence $C(K, Y)$ is an almost central subspace of $C(K, X)$.

For a central subspace $Y$ of a Banach space $X$ and for a compact Hausdorff space $K$, it is not known whether $C(K, Y)$ is a central subspace of $C(K, X)$. But if $C(K, Y) \in(\mathrm{GC})$ and $Y$ is almost central in $X$, then, by Proposition 36 and Theorem 13, $C(K, Y)$ is a central subspace of $C(K, X)$. Now for a Banach space $X$, Theorem 3.6 of [8] gives a sufficient condition for $C(K, X)$ to be in the class $(\mathrm{GC})$. Precisely, if $X$ is a polyhedral Banach space such that $X \in(\mathrm{GC})$ and $\left\{f \in B_{X^{*}}\right.$ : $f(x)=1\} \bigcap \operatorname{ext}\left(B_{X^{*}}\right)$ is finite for each $x \in X$ with $\|x\|=1$, then $C(K, X) \in(\mathrm{GC})$ (by $\operatorname{ext}\left(B_{X^{*}}\right)$, we denote the set of all extreme points of $B_{X^{*}}$ and a Banach space is called polyhedral if the unit ball of each of its finite dimensional subspace is a polytope). In particular, by [8, Fact 1.3(e)], if $X$ is a finite dimensional polyhedral space, then $C(K, X) \in(\mathrm{GC})$. This information together with Proposition 36 give the following corollary.

Corollary 37. Let $Y$ be an almost central subspace of a Banach space $X$ and let $K$ be a compact Hausdorff space. If $Y$ is a polyhedral Banach space such that $Y \in(G C)$ and $\left\{g \in B_{Y^{*}}\right.$ : $g(y)=1\} \bigcap \operatorname{ext}\left(B_{Y^{*}}\right)$ is finite for each $y \in Y$ with $\|y\|=1$, then $C(K, Y)$ is a central subspace of $C(K, X)$. In particular, if $Y$ is a finite dimensional polyhedral central subspace of $X$, then $C(K, Y)$ is a central subspace of $C(K, X)$.

We now discuss the stability problem in injective tensor product spaces.

Proposition 38. Let $K$ be a compact Hausdorff space and let $A$ be an almost central subspace of $C(K)$. Then, for any Banach space $X$, the injective tensor product $A \stackrel{\vee}{\otimes} X$ is almost central in $C(K, X)$.

Proof. Since $A$ is an almost central subspace of $C(K)$, by Proposition 15, $A$ is an $L_{1}$-predual. Then, by [15, Proposition 1], $A$ is an ideal in $C(K)$. Hence, by [15, Lemma 2], $A \stackrel{\vee}{\otimes} X$ is an ideal in $C(K) \stackrel{\vee}{\otimes} X$. Since $C(K, X)=C(K) \stackrel{\vee}{\otimes} X$ (up to an isometry), by Lemma $10, A \mathscr{\vee} X$ is almost central in $C(K, X)$.

Theorem 39. Let $K$ be a compact Hausdorff space and let $A$ be an almost central subspace of $C(K)$. If $Y$ is an almost central subspace of a Banach space $X$, then the injective tensor product 
$A \bigotimes^{\vee} Y$ is an almost central subspace of $C(K) \stackrel{\vee}{\otimes} X$. In particular, $A \stackrel{\vee}{\otimes} Y$ is an almost central subspace of $A \stackrel{\vee}{\otimes} X$.

Proof. By Proposition 38, $A \stackrel{\vee}{\bigotimes} Y$ is almost central in $C(K) \otimes Y_{\vee}=C(K, Y)$. Then, by Proposition 36 and Remark $9, A \bigotimes^{\vee} Y$ is an almost central subspace of $C(K, X)=$ $C(K) \stackrel{\vee}{\otimes} X$. Since $A \stackrel{\vee}{\otimes} Y \subseteq A \stackrel{\vee}{\otimes} X \subseteq C(K, X), A \stackrel{\vee}{\otimes} Y$ is an almost central subspace of $A \otimes X$.

Corollary 40. Let $Z$ be an $L_{1}$-predual. Then, for any almost central subspace $Y$ of a Banach space $X$, the injective tensor product $Z \stackrel{\vee}{\otimes} Y$ is an almost central subspace of $Z \stackrel{\vee}{\otimes} X$.

Proof. Since $Z$ is an $L_{1}$-predual, by [15, Proposition 1], $Z$ is isometric to an ideal in $C(K)$ for some compact Hausdorff space $K$. Then, by Lemma $10, Z$ is an almost central subspace of $C(K)$ (up to an isometry). Therefore, by Theorem $39, Z \stackrel{\vee}{\otimes}$ $Y$ is an almost central subspace of $Z \stackrel{\vee}{\otimes} X$.

We now answer a question raised in [2] and also improve Theorem 6 of [11].

Proposition 41. Let $\Gamma$ be an index set and let $X_{\alpha} \quad(\alpha \in \Gamma)$ be Banach spaces. Then $\bigoplus_{c_{0}} X_{\alpha}$ is a central subspace of $\bigoplus_{\infty} X_{\alpha}$.

Proof. Let $x \in \bigoplus_{\infty} X_{\alpha}$ and $y_{1}, \ldots, y_{n} \in \bigoplus_{c_{0}} X_{\alpha}$. Let $r=$ $\min _{1 \leq i \leq n}\left\|x-y_{i}\right\|$. Since $y_{1}, \ldots, y_{n} \in \bigoplus_{c_{0}} X_{\alpha}$, there exists a finite set $A$ such that $\left\|y_{i}(\alpha)\right\| \leq r$ whenever $\alpha \notin A$.

Define $z \in \bigoplus_{c_{0}} X_{\alpha}$ as

$$
z(\alpha)= \begin{cases}x(\alpha), & \text { if } \alpha \in A, \\ 0, & \text { if } \alpha \notin A\end{cases}
$$

Now for $1 \leq i \leq n$,

if $\alpha \in A$, then $\left\|z(\alpha)-y_{i}(\alpha)\right\|=\left\|x(\alpha)-y_{i}(\alpha)\right\| \leq\left\|x-y_{i}\right\|$ and

if $\alpha \notin A$, then $\left\|z(\alpha)-y_{i}(\alpha)\right\|=\left\|y_{i}(\alpha)\right\| \leq r \leq\left\|x-y_{i}\right\|$.

Hence $\left\|z-y_{i}\right\| \leq\left\|x-y_{i}\right\|$ for all $i$.

Corollary 42. The class $(G C)$ is stable under $c_{0}$-direct sum of Banach spaces.

Proof. If $X_{\alpha} \in$ (GC) for all $\alpha \in \Gamma$, then, by [2, Theorem 4.7] and Proposition $41, \bigoplus_{\mathcal{C}_{0}} X_{\alpha}$ is a central subspace of $\bigoplus_{\infty} X_{\alpha}^{* *}=\left(\bigoplus_{c_{0}} X_{\alpha}\right)^{* *}$. Hence the result follows.

In [8], Veselý defined a new direct sum called polyhedral direct sum. We now prove the stability of some ball intersection properties under polyhedral direct sums.

Definition 43 (see [8]). A norm $\pi$ on $\mathbb{R}_{+}^{n}$ is called polyhedral if it is of the form $\pi(t)=\max _{1 \leq j \leq m} g_{j}(t)$, where $g_{1}, \ldots, g_{m} \in$ $\left(\mathbb{R}^{n}\right)^{*}$. In this case, we say that the family $\left\{g_{1}, \ldots, g_{m}\right\}$ generates $\pi$. Now Lemma 1.5 of [8] shows that if $\left\{g_{1}, \ldots, g_{m}\right\}$ is a minimal family generating $\pi$, then $g_{j}(i) \geq 0$ for all $i=1, \ldots, n$ and $j=1, \ldots, m$.

We say that a Banach space $X$ is the polyhedral direct sum of Banach spaces $X_{1}, \ldots, X_{n}$ if $X=X_{1} \oplus \cdots \oplus X_{n}$ and the norm on $X$ is of the form $\|x\|_{X}=\pi(\|x(1)\|, \ldots,\|x(n)\|)$, where $\pi$ is a polyhedral nondecreasing norm on $\mathbb{R}_{+}^{n}$. In this case, we write $X=\left(X_{1} \oplus \cdots \oplus X_{n}\right)_{\pi}$.

Our next theorem proves that the property of being a central subspace is stable under polyhedral direct sums.

For $n \in \mathbb{N}$ and $1 \leq i \leq n$, we denote by $e_{i}$ the $i$ th canonical unit vector of $\mathbb{R}^{n}$.

Theorem 44. Let $X$ be a polyhedral direct sum of Banach spaces $X_{i}(1 \leq i \leq n)$ and let $Y_{i}$ be a subspace of $X_{i}(1 \leq i \leq$ $n)$. Let $\pi$ be the corresponding polyhedral norm and suppose $\pi\left(e_{i}\right) \neq 0$ for all $i$. Then the polyhedral sum $Y$ of $Y_{i}(1 \leq i \leq n)$ is a central subspace of $X$ if and only if $Y_{i}$ is a central subspace of $X_{i}$ for all $i$.

Proof. Suppose $Y$ is a central subspace of $X$. Fix an $m \in$ $\{1, \ldots, n\}$. Let $x_{m} \in X_{m}$ and $y_{m, k} \in Y_{m}(1 \leq k \leq p)$. Define $x \in X$ and $y_{k} \in Y(1 \leq k \leq p)$ as

$$
x(i)=\left\{\begin{array}{ll}
x_{m}, & \text { if } m=i, \\
0, & \text { otherwise, }
\end{array} \quad y_{k}(i)= \begin{cases}y_{m, k}, & \text { if } m=i, \\
0, & \text { otherwise }\end{cases}\right.
$$

Then there exists a $y \in Y$ such that $\left\|y-y_{k}\right\|_{X} \leq\left\|x-y_{k}\right\|_{X}$ for $1 \leq k \leq p$. Therefore, for $1 \leq k \leq p$, we have

$$
\begin{aligned}
\| y(m) & -y_{k}(m) \| \pi\left(e_{m}\right) \\
& =\pi\left(\left\|y(m)-y_{k}(m)\right\| e_{m}\right) \\
& \leq \pi\left(\left\|y(1)-y_{k}(1)\right\|, \ldots,\left\|y(n)-y_{k}(n)\right\|\right) \\
& \leq \pi\left(\left\|x(1)-y_{k}(1)\right\|, \ldots,\left\|x(n)-y_{k}(n)\right\|\right) \\
& =\left\|x(m)-y_{k}(m)\right\| \pi\left(e_{m}\right) .
\end{aligned}
$$

Since $\pi\left(e_{i}\right) \neq 0$ for all $i$, we get $\left\|y(m)-y_{m, k}\right\| \leq\left\|x(m)-y_{m, k}\right\|$ for $1 \leq k \leq p$. Hence $Y_{m}$ is a central subspace of $X_{m}$.

Conversely suppose $Y_{i}$ is central in $X_{i}$ for all $i$. Let $x \in X$ and $y_{k} \in Y(1 \leq k \leq p)$. Then, for $1 \leq m \leq n$, there exists a $y_{m} \in Y_{m}$ such that $\left\|y_{m}-y_{k}(m)\right\| \leq\left\|x(m)-y_{k}(m)\right\|$ for $1 \leq k \leq p$. Define $y \in Y$ as $y(i)=y_{i}(1 \leq i \leq n)$. Now, by the monotonicity of $\pi$,

$$
\begin{aligned}
\left\|y-y_{k}\right\|_{X} & \leq \pi\left(\left\|\left(x-y_{k}\right)(1)\right\|, \ldots,\left\|\left(x-y_{k}\right)(n)\right\|\right) \\
& =\left\|x-y_{k}\right\|_{X} \quad \text { for } 1 \leq k \leq p .
\end{aligned}
$$

Hence $Y$ is a central subspace of $X$.

An argument similar to the one used to prove Theorem 44 gives the following. 
Theorem 45. Let $X$ be a polyhedral direct sum of Banach spaces $X_{i}(1 \leq i \leq n)$ and let $Y_{i}$ be a subspace of $X_{i}(1 \leq i \leq$ $n)$. Let $\pi$ be the corresponding polyhedral norm and suppose $\pi\left(e_{i}\right) \neq 0$ for all $i$. Then the polyhedral sum $Y$ of $Y_{i}(1 \leq i \leq n)$ is an AC-subspace of $X$ if and only if $Y_{i}$ is an AC-subspace of $X_{i}$ for all $i$.

\section{Acknowledgments}

The author would like to thank Professor T. S. S. R. K. Rao for many helpful discussions and valuable suggestions. The author also thanks the referees for their extensive comments that lead to an improved version of the paper.

\section{References}

[1] L. Veselý, "Generalized centers of finite sets in Banach spaces," Acta Mathematica Universitatis Comenianae, vol. 66, no. 1, pp. 83-115, 1997.

[2] P. Bandyopadhyay and T. S. S. R. K. Rao, "Central subspaces of Banach spaces," Journal of Approximation Theory, vol. 103, no. 2, pp. 206-222, 2000.

[3] J. Diestel and J. J. Uhl, Jr., Vector Measures, vol. 15 of Mathematical Surveys, American Mathematical Society, Providence, RI, USA, 1977.

[4] P. Bandyopadhyay and S. Dutta, "Almost constrained subspaces of Banach spaces," Proceedings of the American Mathematical Society, vol. 132, no. 1, pp. 107-115, 2004.

[5] P. Bandyopadhyay and S. Dutta, "Almost constrained subspaces of Banach spaces. II," Houston Journal of Mathematics, vol. 35, no. 3, pp. 945-957, 2009.

[6] G. Godefroy, N. J. Kalton, and P. D. Saphar, "Unconditional ideals in Banach spaces," Studia Mathematica, vol. 104, no. 1, pp. 13-59, 1993.

[7] P. Harmand, D. Werner, and W. Werner, M-ideals in Banach Spaces and Banach Algebras, vol. 1547 of Lecture Notes in Mathematics, Springer, Berlin, Germany, 1993.

[8] L. Veselý, "Polyhedral direct sums of Banach spaces, and generalized centers of finite sets," Journal of Mathematical Analysis and Applications, vol. 391, no. 2, pp. 466-479, 2012.

[9] A. Lima, "The metric approximation property, norm-one projections and intersection properties of balls," Israel Journal of Mathematics, vol. 84, no. 3, pp. 451-475, 1993.

[10] J. Lindenstrauss, "Extension of compact operators," Memoirs of the American Mathematical Society, vol. 48, p. 112, 1964.

[11] T. S. S. R. K. Rao, "Central and almost constrained subspaces of Banach spaces," Operators and Matrices, vol. 6, no. 2, pp. 371383, 2012.

[12] A. Lima, "Intersection properties of balls and subspaces in Banach spaces," Transactions of the American Mathematical Society, vol. 227, pp. 1-62, 1977.

[13] H. E. Lacey, The Isometric Theory of Classical Banach Spaces, Springer, New York, NY, USA, 1974, Die Grundlehren der Mathematischen Wissenschaften, Band 208.

[14] M. Baronti, "Norm-one projections onto subspaces of $l^{\infty}$," Archiv der Mathematik, vol. 51, no. 3, pp. 242-246, 1988.

[15] T. S. S. R. K. Rao, "On ideals in Banach spaces," The Rocky Mountain Journal of Mathematics, vol. 31, no. 2, pp. 595-609, 2001. 


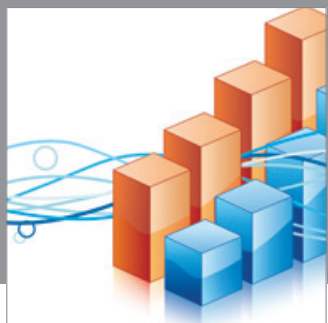

Advances in

Operations Research

mansans

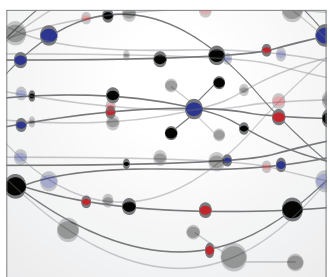

The Scientific World Journal
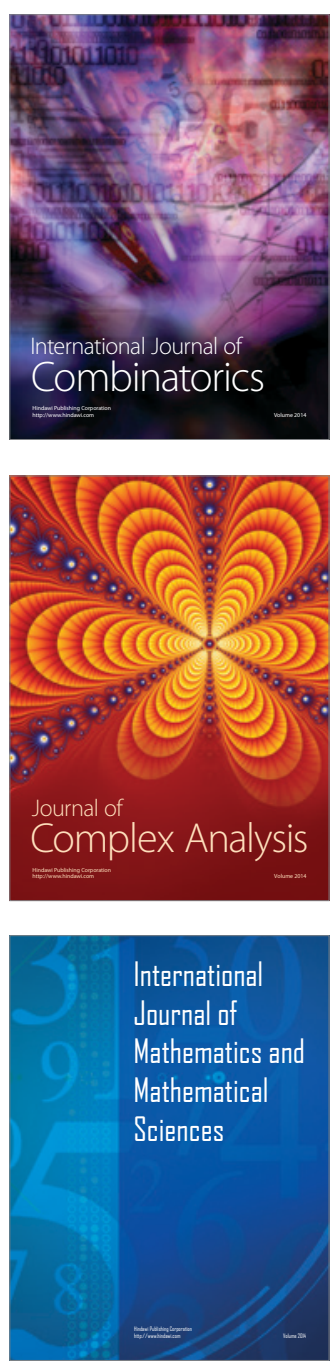
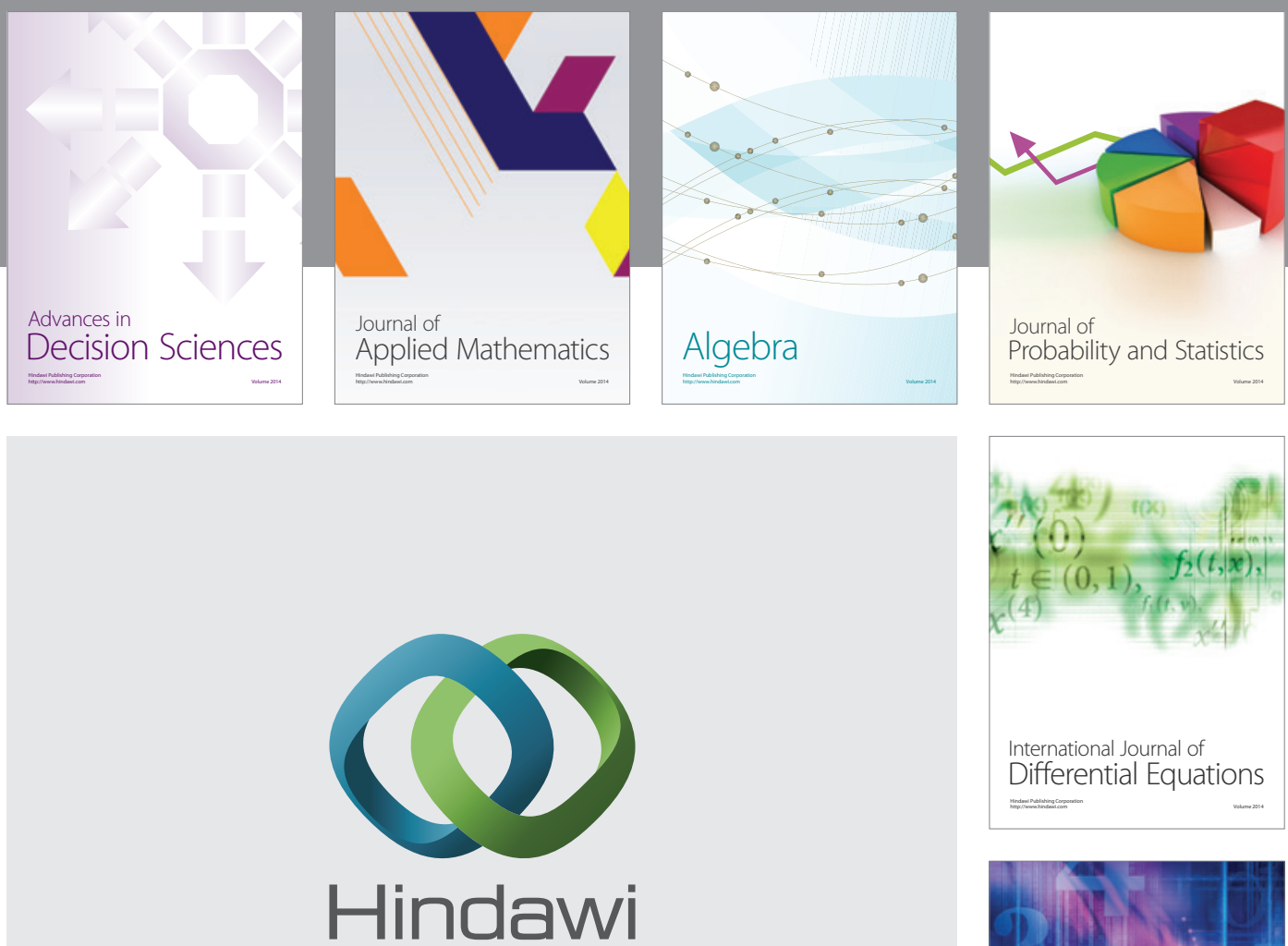

Submit your manuscripts at http://www.hindawi.com
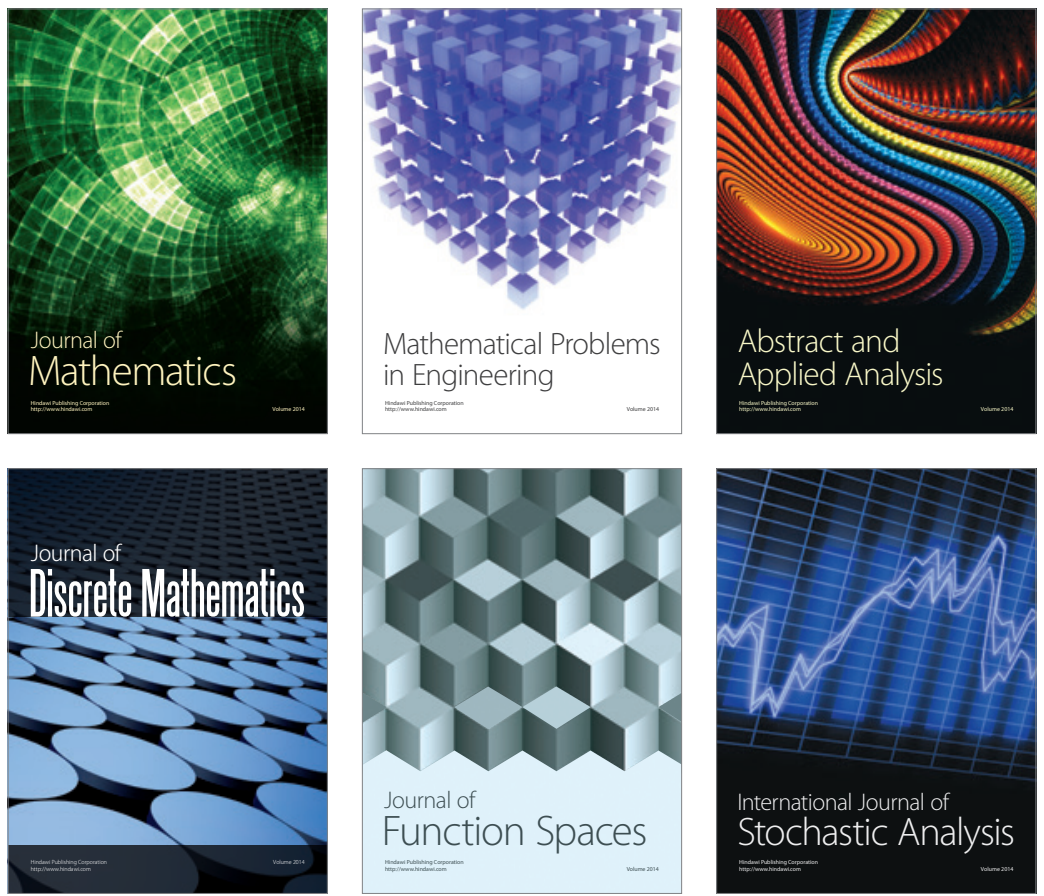

Journal of

Function Spaces

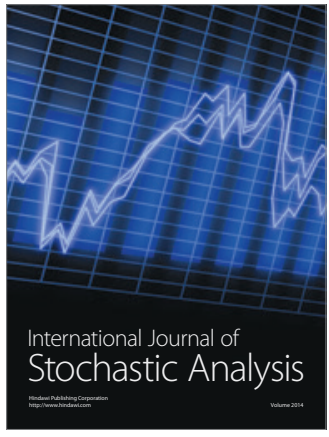

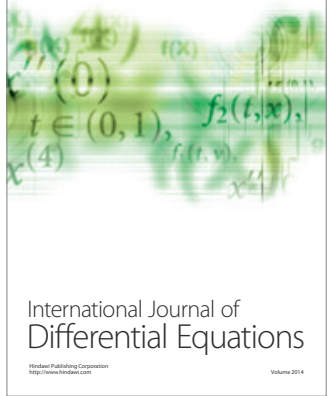
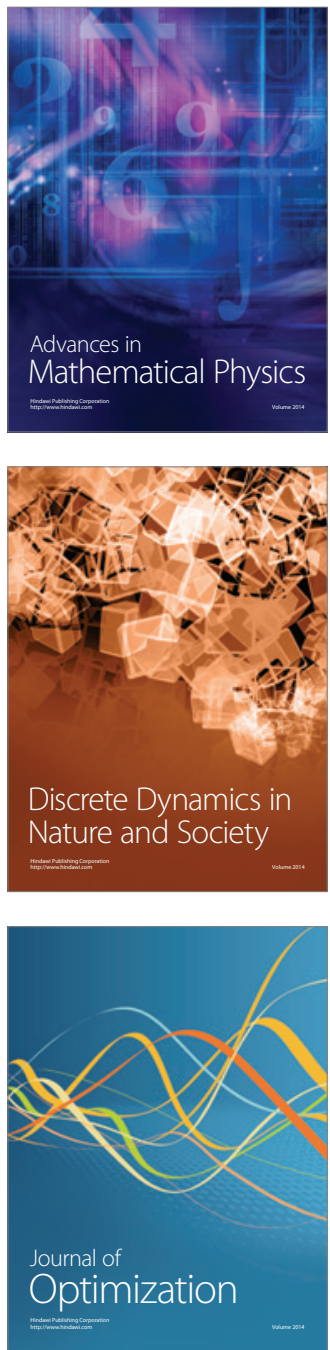